Introduction Non-alcoholic fatty liver disease (NAFLD) is the leading liver disease in Europe and North America. 30\% of patients with NAFLD are estimated to develop inflammatory non-alcoholic steatohepatitis (NASH) with the potential to lead to cirrhosis and hepatocellular carcinoma. Current evidence suggests that gut bacterial products can drive hepatic inflammation by activating specific innate pattern recognition receptors (PRRs) such as TLR4 and the NALP3 inflammasome. Both receptors are expressed by liver resident macrophages, Kupffer cells (KCs).

Methods To determine the role of KCs and interactions with PRRs in NASH, mice were fed a methionine choline deficient (MCD) diet for three weeks to induce NASH. Liposomal clodronate was used to deplete KCs. Serum ALT levels were measured and hepatic inflammatory infiltrates characterised by flow cytometry. Real-time qPCR was used to assess changes in gene expression. Murine findings were correlated with human liver tissue from NASH patients. Groups were compared by one-way ANOVA and significance set at $\mathrm{P}<0.05$.

Results NALP3 KO (knock-out), TLR4 KO or KC deficient WT mice on a MCD diet developed reduced liver damage and decreased T lymphocyte recruitment compared to WT MCD controls. Combined KC depletion and NLRP3 KO however lead to significantly worse liver injury and progressive fibrosis as measured by collagen expression. Further investigation revealed an as yet unrecognised role for KC expressed NALP6 activation which mediated antiinflammatory responses and modulation of hepatic IL-22 responsiveness to reduce liver injury.

Conclusion

1. KC activation through NALP3 and TLR4 increases hepatic inflammation in the MCD model of NASH.

2. KCs have a dual role in NASH as they also express NALP6 with anti-inflammatory properties and are able to reduce hepatic injury through modulation of IL-22 responsiveness modulation.

Disclosure of Interest None Declared

\section{Endoscopy free papers}

\section{OC-009 ENDOSCOPIC THERAPY FOR ZENKER'S DIVERTICULUM - A "BRIDGE" TOO FAR FOR GASTROENTEROLOGISTS?}

doi:10.1136/gutjnl-2013-304907.009

1."M Smith, 'M Widlak, 'N Molony, 'S Ishaq. 'Dudley Group of Hospitals NHS Foundation Trust, NHS, Dudley, UK

Introduction Zenker's diverticulum, caused by dysfunction of the cricopharyngeal (CP) muscle, is a disease of the elderly causing dysphagia. Although rigid endoscopic CP myotomy is the treatment of choice, flexible endoscopic therapy is another technique available. It is performed under sedation without need for anaesthetic or neck extension, a key advantage in elderly patients with significant comorbidity.
Methods We describe the first UK experience of endoscopic CP myotomy for Zenker's diverticulum.

Results 4 patients ( 3 male) were referred, aged 74, 73, 60, 80 respectively, with proven Zenker's diverticulum on barium radiology and characteristic symptomatology. Zenker length was $7.0,4.0,2.5,2.5 \mathrm{~cm}$ respectively. 2 patients were refused surgery due to cardiovascular comorbidity, 1 due to limited neck extension with 1 case personal preference. The procedure was performed using propofol sedation. The muscle bridge was cut by hook knife electrocautery alone (3) or with Argon (1). A supplementary clip was applied post electrocautery in 1 case. All patients were observed for 24 hours for signs of perforation and then discharged. There were no complications.

At 4 weeks 3 patients had complete resolution of their symptoms, 1 with marked improvement. Follow up time to date 3.4.7.11 months respectively. There was no reported recurrence of symptoms.

Conclusion Diverticulotomy with a flexible endoscope is an effective treatment for Zenker's diverticulum. It is a relatively simple yet under-utilised technique that avoids general anaesthesia in elderly/ high-risk patients.

Disclosure of Interest None Declared

\section{OC-010 LARGE COHORT STUDY EVALUATING THE ROLE OF HYBRID ESD (H-ESD) AND CONVENTIONAL PIECEMIEAL EMR TECHNIQUE IN THE RESECTION OF LARGE AND CHALLENGING COLONIC POLYPS DEMONSTRATES NO OUTCOME BENEFIT OF H-ESD OVER EMR}

doi:10.1136/gutjnl-2013-304907.010

'R Bhattacharyya, 'P Basford, 'S Tholoor, ' $G$ Longcroft-Wheaton, 1,* $\mathrm{P}$ Bhandari. ${ }^{1}$ Gastroenterology, Portsmouth Hospitals NHS Trust, Portsmouth, UK

Introduction The learning curve for ESD in the west is very long, so a hybrid technique has been proposed. The impact of Hybrid ESD (H-ESD) technique on clinical outcome is unclear. We aim to compare the outcome benefits of Multi-piece EMR and H-ESD in the resection of challenging colonic polyps.

Methods A Prospective cohort study of endoscopic resection of difficult colonic polyps. Patients were tertiary referrals from experienced endoscopists. EMR was defined as submucosal injection followed by piecemeal snare resection. H-ESD involved submucosal injection before mucosal incision with an ESD knife followed by snare resection of the lesion. Endoscopic follow up was performed. Multiple linear regression analysis was performed using SPSS.

Results $347 \mathrm{flat} / \mathrm{sessile} \mathrm{polyps}>20 \mathrm{~mm}$ were resected between 2007-12. Mean follow-up was 1004 days.

H-ESD Cohort $\mathrm{N}=110 / 347(32 \%)$. Mean size was $45 \mathrm{~mm}$ (range 10-170). 25/110(23\%) were salvage procedures for scarred lesions due to failed EMR attempts by other endoscopists. Endoscopic clearance was achieved in $95.5 \%$ of procedures. Need for surgery $(n=4)$ : 1 for perforation and 3 for unexpected cancer. 98.6\% showed no evidence of recurrence at endoscopic follow up.

EMR cohort $\mathrm{N}=237 / 347(68 \%)$. Mean size was $42 \mathrm{~mm}$ (range 20-150). 11/237(4.6\%) were salvage procedures for polyps with

Abstract 0C-010 Table

\begin{tabular}{|c|c|c|c|c|c|c|c|c|}
\hline & SIZE & & TECHNIQUE & & PREV EMR & & SITE & \\
\hline \multirow{2}{*}{$\begin{array}{l}\text { COMPLICATIONS } \\
\text { (25) }\end{array}$} & $15 / 271(5.5 \%)$ & $\begin{array}{l}8 / 76 \\
(10.5 \%)\end{array}$ & $11 / 110(10 \%)$ & $12 / 237(5 \%)$ & $3 / 36(8 \%)$ & $\begin{array}{l}20 / 311 \\
(6 \%)\end{array}$ & $20 / 250(8 \%)$ & $3 / 97$ (3\% \\
\hline & $P=0.016$ & & $P=0.11$ & & $P=0.521$ & & $P=0.201$ & \\
\hline $\begin{array}{l}\text { RECURRENCE } \\
(35)\end{array}$ & $P=0.0001$ & & $P=0.156$ & & $P=0.0001$ & & $P=0.105$ & \\
\hline
\end{tabular}

